\title{
Analysis of bio-anode performance through electrochemical impedance spectroscopy
}

\author{
Annemiek ter Heijne ${ }^{\mathrm{a}, 1}$, Olivier Schaetzle ${ }^{\mathrm{b}, 1}$, Sixto Gimenez ${ }^{\mathrm{c}}$, Lucia Navarro ${ }^{\mathrm{c}}$, \\ Bert Hamelers ${ }^{\text {b }}$, Francisco Fabregat-Santiago ${ }^{c, *}$ \\ a Sub-department of Environmental Technology, Wageningen University, Bornse Weilanden 9, P.O. Box 17, 6700 AA Wageningen, The Netherlands \\ ${ }^{\mathrm{b}}$ Wetsus, Centre of Excellence for Sustainable Water Technology, Oostergoweg 9, P.O. Box 1113, 8900 CC Leeuwarden, The Netherlands \\ c Photovoltaic and Optoelectronics Devices Group, Departament de Física, Universitat Jaume, Av. Sos Baynat s/n, 12006 Castelló de la Plana, Spain
}

\section{A R T I C L E I N F O}

\section{Article history:}

Received 27 November 2014

Received in revised form 29 March 2015

Accepted 1 April 2015

Available online xxxx

\section{Keywords:}

Electrochemical impedance spectroscopy

Internal resistance

Microbial fuel cell

Charge transfer

Diffusion

\begin{abstract}
A B S T R A C T
In this paper we studied the performance of bioanodes under different experimental conditions using polarization curves and impedance spectroscopy. We have identified that the large capacitances of up to $1 \mathrm{mF} \cdot \mathrm{cm}^{-2}$ for graphite anodes have their origin in the nature of the carbonaceous electrode, rather than the microbial culture.

In some cases, the separate contributions of charge transfer and diffusion resistance were clearly visible, while in other cases their contribution was masked by the high capacitance of $1 \mathrm{mF} \cdot \mathrm{cm}^{-2}$. The impedance data were analyzed using the basic Randles model to analyze ohmic, charge transfer and diffusion resistances. Increasing buffer concentration from 0 to $50 \mathrm{mM}$ and increasing $\mathrm{pH}$ from 6 to 8 resulted in decreased charge transfer and diffusion resistances; lowest values being $144 \Omega \cdot \mathrm{cm}^{2}$ and $34 \Omega \cdot \mathrm{cm}^{2}$, respectively. At acetate concentrations below $1 \mathrm{mM}$, current generation was limited by acetate. We show a linear relationship between inverse charge transfer resistance at potentials close to open circuit and saturation (maximum) current, associated to the ButlerVolmer relationship that needs further exploration.
\end{abstract}

(c) 2015 Elsevier B.V. All rights reserved.

\section{Introduction}

In the search for renewable energy sources, Microbial Electrochemical Systems (MESs) offer new and exciting ways to convert chemical energy into electrical energy, or vice versa. In MESs, micro-organisms or enzymes are used to catalyze electrochemical reactions at the anode or the cathode in either a microbial fuel cell (MFC) when electricity is recovered, or a microbial electrolysis cell (MEC) when electricity is used to drive the conversion of electrical energy into chemical energy [1,2].

The combination of electrodes and microorganisms, and the related bioelectrochemical reactions, pose a challenge on the understanding of the systems and the reactions that determine performance of MESs. In order to analyze their behavior, several electrochemical tools are common in MES research [1], for example chronoamperic measurements to determine polarization curves, and Electrochemical Impedance Spectroscopy (EIS) to study the processes and reactions limiting MES performance [3-5].

EIS is a method that allows investigating the electrical properties of a system by measuring its response in current or voltage after imposing a small AC perturbation (in voltage or current) to a stationary state and

\footnotetext{
* Corresponding author.

E-mail address: fabresan@fca.uji.es (F. Fabregat-Santiago).

${ }^{1}$ Both authors contributed equally to this work
}

obtaining the impedance through $Z=d V / d I$ [6]. The measurement is performed in a wide range of frequencies, which reveals the different characteristic relaxation times related to the processes and reactions occurring in the different elements that compose electrodes or complete devices, at their operating conditions. The obtained impedance spectra allow interpretation of the electrochemical characteristics of the system through the use of the appropriate equivalent electrical circuit model. These models are in general well-defined for systems in which parameters are well-known and controlled (e.g. clean electrode surface, pure electrolytes). For MESs, however, models are less straightforward because MESs are complex systems involving a biofilm (often of unknown structure) interacting with the electrodes through not well-established mechanisms. Additionally, the relationship between the substrate and the redox activity is interfaced by the microbial metabolism, which increases the complexity of the models traditionally used [7-9].

In a recent review article [3], the lack of EIS studies in terms of appropriate equivalent circuits that describe the physical-chemical parameters of MES, were addressed. In the same direction, we showed in a previous study and through a systematical experimental approach that the use of traditional models was leading to an underestimation of the diffusion parameter [5]. While for oxygen reducing biocathodes, diffusion of oxygen was in many cases the dominant limiting factor [5, $10]$ other processes may be determining the performance of bioanodes. In the present study, we analyze the performance of a bioanode using 
EIS, using a systematic experimental approach by changing the key operation parameters substrate concentration, buffer concentration, and $\mathrm{pH}$.

\section{Materials \& methods}

\subsection{Electrochemical cell setup}

A flow-through bio-electrochemical cell was used, containing two flow channels with a projected surface area of $22 \mathrm{~cm}^{2}$ and a channel depth of $1.5 \mathrm{~cm}$ [11]. The flow channels were separated by a cation exchange membrane (Fumasep FTCM-E, Fumatech, Braunschweig, Germany). Both compartments contained $\mathrm{Ag} / \mathrm{AgCl}, 3 \mathrm{M} \mathrm{KCl}$, reference electrodes $(+205 \mathrm{mV}$ vs NHE) inserted in through a top hole in the middle of the flow compartment, see scheme 1(a). The anode and the cathode were porous graphite plates (Müller \& Rössner GmbH \& Co, Troisdorf, Germany) as shown in Figure S1 in supporting information. For comparison, a fluorine doped tin oxide flat plate (Xop Fisica, Spain) was also used as anode.

Scheme 1 presents a picture and a schematic view of the cell.

\subsection{Electrochemical cell operation and experimental conditions}

The cell was inoculated on day 1 with $100 \mathrm{~mL}$ of anolyte from an already working MFC that was running on acetate. The anolyte had a total volume of $1 \mathrm{~L}$ and consisted of phosphate buffer $(\mathrm{pH}=7,0.02 \mathrm{M})$, and macro- and micro-nutrients ( $10 \mathrm{~mL} / \mathrm{L}$ and $1 \mathrm{~mL} / \mathrm{L}$ ) as described in a previous work [11]. The catholyte also had a total volume of $1 \mathrm{~L}$ and was a $0.05 \mathrm{M}$ potassium ferricyanide solution in $0.02 \mathrm{M}$ phosphate buffer at $\mathrm{pH}=7$.

The bioanode was started up with an external load of $1000 \Omega$ that was progressively lowered to $250 \Omega$. Once the cell reached satisfying performances (after 24 days), cell potential of $0.59 \mathrm{~V}$; anode potential of $-0.455 \mathrm{~V} \mathrm{vs} \mathrm{Ag} / \mathrm{AgCl}$; current of $1.2 \mathrm{~A} / \mathrm{m}^{2}$ with respect to membrane surface area; power of $695 \mathrm{~mW} / \mathrm{m}^{2}$ or $42.4 \mathrm{~W} / \mathrm{m}^{3}$, the cell was considered to be started-up and electrochemical analyses were performed. The flow rate was $12 \mathrm{~L} / \mathrm{h}$, resulting in a linear flow speed of $1.1 \mathrm{~cm} / \mathrm{s}$. All experiments were performed in a temperature controlled chamber at $30^{\circ} \mathrm{C}$.

The MFC was started up at acetate concentration of $5 \mathrm{mM}$, phosphate buffer concentration of $20 \mathrm{mM}$ and $\mathrm{pH}$ 7. This configuration was characterized electrochemically both using chronoamperometry to determine polarization curves, and impedance spectroscopy at different bias potentials. Randles equivalent circuit was proposed to fit data and identify parameters dominating electrical behavior at bioanode. Then, acetate concentration, buffer concentration, and $\mathrm{pH}$ values were changed one by one, while keeping constant the rest of the parameters. The tested conditions were: acetate concentration: $0 \mathrm{mM}, 0.5 \mathrm{mM}$,

(a)

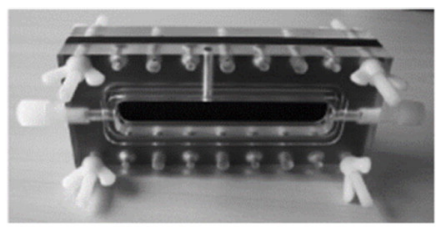

(b)

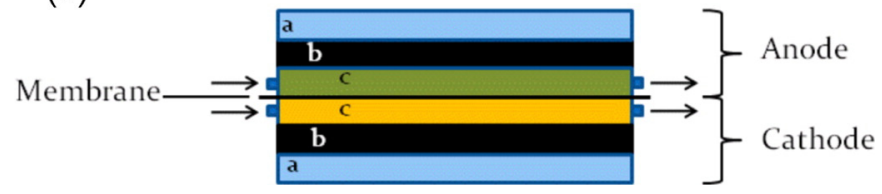

Scheme 1. Cell design. (a) View of one half of the cell with the flow channel in the front. (b) Schematic view of the whole cell (a. Plexiglas support; b. flat graphite plate; $c$. (green) anodic flow compartment; (yellow) cathodic flow compartment).
$1 \mathrm{mM}, 5 \mathrm{mM}, 20 \mathrm{mM}$, buffer concentration: $0 \mathrm{mM}, 20 \mathrm{mM}, 50 \mathrm{mM}$, $100 \mathrm{mM}$, and pH: 6, 7 and 8. Linear flow velocity was kept constant at $1.1 \mathrm{~cm} / \mathrm{s}$. These conditions were tested always at an anode potential of $-0.44 \mathrm{~V}$ vs $\mathrm{Ag} / \mathrm{AgCl}$ for impedance spectra comparison.

The overall bioelectrochemical oxidation of acetate that provides the electrons to the bioanode, is written as:

$\mathrm{C}_{2} \mathrm{H}_{3} \mathrm{O}_{2}^{-}+4 \mathrm{H}_{2} \mathrm{O} \rightarrow 2 \mathrm{HCO}_{3}^{-}+9 \mathrm{H}^{+}+8 e^{-}$.

\subsection{Electrochemical characterization}

Bare graphite electrode was first measured to identify its electrochemical behavior when immersed in the same solution as the bioanodes. Planar Fluorine doped tin oxide (FTO) was also tested to compare the response of this flat electrode with that of graphite.

For each set of solution parameters, the polarization curve of the bioanode was recorded just before the impedance measurement was started. Current-voltage behavior and impedance spectra of the bioanode were measured using a potentiostat (IVIUM technologies, Eindhoven, The Netherlands) connected to the electrochemical cell described above in three electrode configuration: the bioanode was connected as the working electrode, the carbon cathode was connected as the counter electrode, and the $\mathrm{Ag} / \mathrm{AgCl}$ electrode in the anodic compartment was used as the reference electrode. Impedance spectra were taken at steady state potentials vs $\mathrm{Ag} / \mathrm{AgCl}$ close to or slightly more positive than the open circuit anode potential, after equilibration time of $500 \mathrm{~s}$. Measurement frequencies ranged from $10 \mathrm{kHz}$ to $2 \mathrm{mHz}$ and the amplitude of the AC perturbation was $10 \mathrm{mV}$.

\subsubsection{Model description}

Randles equivalent circuit shown in Fig. 1(b), was used to fit the impedance data obtained and to describe the electrical processes occurring at the bioanode. In this circuit, $R_{S}$ represents a series resistance associated with electrode and bulk electrolyte conductivities, contacts and wires; $R_{c t}$ is a resistance resulting from charge transfer between bioelectrode to the solution; $C_{d l}$ accounts for the capacitance at the interface between solution and bioanode due to charge accumulation. In some cases, instead of a pure capacitor a constant phase element, with $\mathrm{CPE}$ index $>0.8$, was used to fit data. In these cases, equivalent capacitance was calculated through Brug equation [12]. Finally, $Z_{d}$ is a diffusion element describing the transport of active species in the solution given by

$Z_{d}=R_{d} \frac{\tanh \left(j \omega / \omega_{d}\right)^{1 / 2}}{\left(j \omega / \omega_{d}\right)^{1 / 2}}$

where $R_{d}$ is the diffusion resistance, $j=\sqrt{-1}, \omega$ is the (angular) frequency of the measurement and $\omega_{d}=D / L^{2}$ the diffusion frequency, with $D$ the diffusion coefficient and $L$ the thickness of the boundary layer.

\section{Results and discussion}

\subsection{Analysis of bioanode performance at different anode potentials}

The current-voltage $(\mathrm{J}-\mathrm{V})$ curve and impedance spectra of the bioanode at $5 \mathrm{mM}$ acetate and $20 \mathrm{mM}$ buffer at pH 7 are shown in Fig. 1(a).

The polarization curve for the bioanode shows the S-shape typical for bioelectrochemical reactions [7,11], with the current saturating to $1.4 \mathrm{~A} \mathrm{~m}^{-2}$ at an anode potential of $-0.35 \mathrm{~V} \mathrm{vs} \mathrm{Ag} / \mathrm{AgCl}$. Impedance spectra are presented in the Nyquist plot in Fig. 1(c) and (d); additional data can be found in Figure S1 in Supporting Information. At the most negative anode potential of $-0.45 \mathrm{~V} \mathrm{vs} \mathrm{Ag} / \mathrm{AgCl}$, one single deformed arc was observed. At higher anode potentials, two arcs appear instead 


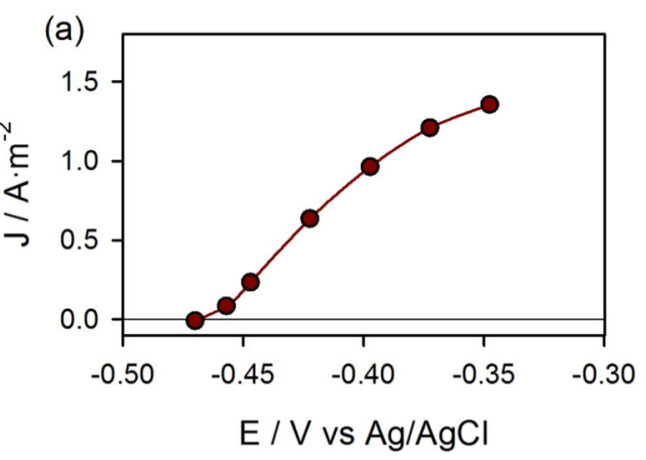

(c)

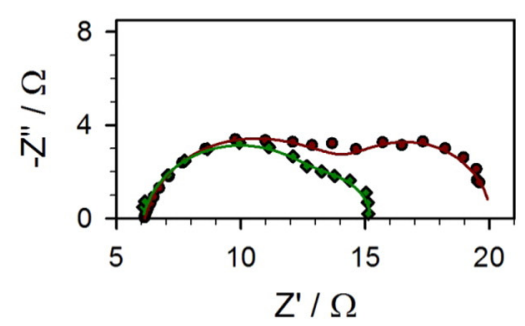

(b)

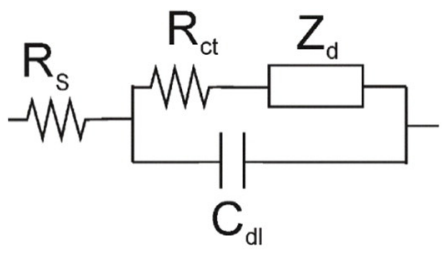

(d)

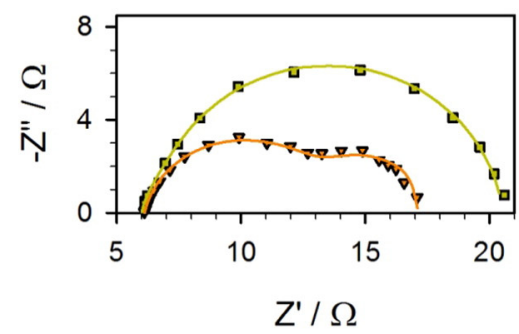

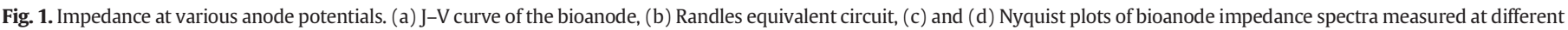

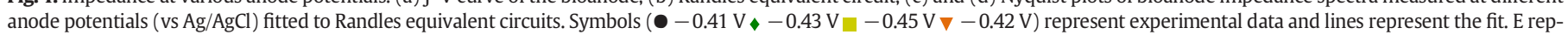
resents bioanode potential.

of one. The appearance of a single arc is often interpreted as a charge transfer resistance, however, in this case it is caused by the presence of a very large capacitance in the bioanode (around $1 \mathrm{mF} \cdot \mathrm{cm}^{-2}$ ), as was also reported in previous work on biocathodes [5]. This very large capacitance may mask the presence of two processes: charge transfer and diffusion resistance, resulting in a single arc that we do observe at potentials higher than $-0.45 \mathrm{~V}$ vs $\mathrm{Ag} / \mathrm{AgCl}$. This effect is illustrated in Fig. 2(a), where the simulated impedance of a Randles circuit using a capacitance of $1 \mathrm{mF} \cdot \mathrm{cm}^{-2}$ (black line) is compared with the simulated impedance provided by the same parameters but smaller, $0.2 \mathrm{mF} \cdot \mathrm{cm}^{-2}$ (red line) and a much smaller, $10 \mu \mathrm{F} \cdot \mathrm{cm}^{-2}$ (green line) capacitances.

At the highest capacitance, we observe one single arc, which is the result of similar characteristic times of the charge transfer and diffusion resistance. At the two lowest capacitances however, the semicircle at

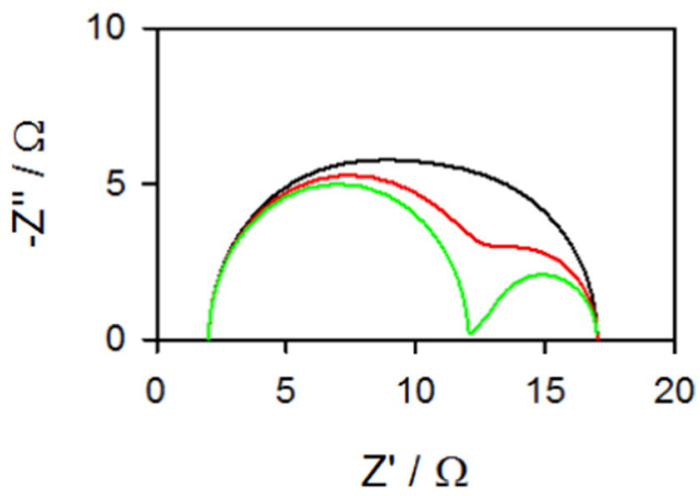

Fig. 2. Simulated Nyquist plots for different capacitances. Impedance spectra of a Randles equivalent circuit are simulated with $R_{\mathrm{s}}=2 \Omega, \mathrm{R}_{\mathrm{ct}}=10 \Omega, \mathrm{R}_{\mathrm{d}}=5 \Omega, \omega_{\mathrm{d}}=0.5 \mathrm{~s}^{-1}$ and $\mathrm{C}_{\mathrm{dl}}=1.0 \mathrm{mF} \cdot \mathrm{cm}^{-2}$ (black line), $0.2 \mathrm{mF} \cdot \mathrm{cm}^{-2}$ (red line), and $0.01 \mathrm{mF} \cdot \mathrm{cm}^{-2}$ (green line). For low anode double layer capacitances, the arcs associated to charge transfer (high frequency, hf) and diffusion (low frequency, lf) may be clearly distinguished. However, for high $C_{d l}$, the two arcs merge. (For interpretation of the references to color in this figure legend, the reader is referred to the web version of this article.) high frequencies associated to charge transfer and interfacial accumulation is clearly separated from the low frequency diffusion process. Measurement of the bare carbon electrodes showed indeed a capacitance of $\sim 1 \mathrm{mF} \cdot \mathrm{cm}^{-2}$ (Figure S2 in Supporting Information). This is 100 times larger than that of a non-capacitive, flat fluorine doped tin oxide (FTO) electrode.

The large capacitance of the carbon electrode has two origins: on the one side, the surface of the anodes presents micro and nanoporosity (Figure S3 in Supporting Information). Porosity of the electrode increases the roughness, and thus the double layer capacitance. On the other side, ion intercalation in the graphite atomic layers produces and extra increase of electrode capacitance. The combination of these two contributions has been known for a long time and has been used to build supercapacitors and battery electrodes [13-16]. In our specific case, microporosity provides a large area accessible for the growth of an electrochemically active biofilm, which is needed to obtain large currents.

Taking these considerations into account, impedance in data of the bioanode at different potentials as in Fig. 1(c) and (d) was fitted to the Randles equivalent circuit model (Fig. 1(b)). Values obtained from the fits are given in Fig. 3.

Capacitance data in Fig. 3(a) match very well with those obtained for the bare graphite electrode and also with data reported in previous paper analyzing impedance of an oxygen reducing biocathode on the same type of electrode [5], with values around $1 \mathrm{mF} \mathrm{cm}^{-2}$. This result demonstrates that a possible contribution of the microbial biofilm to bioanode capacitance in this case is negligible with respect to the capacitance of the graphite electrode.

All measurements were performed close to open circuit anode potential, to measure in the region in which bioanode performance was stable. At the most negative anode potentials, $R_{c t}$ dominates the total resistance of the bioanode impedance (Fig. $3(\mathrm{~b}))$. $R_{c t}$ presents a minimum around $-0.42 \mathrm{~V} v \mathrm{Ag} / \mathrm{AgCl}$ and starts to rise when moving to more positive anode potentials. $R_{d}$ decreases as the anode potential is more negative. This is the same behavior as found for biocathodes [5], which suggests that charge transfer and transport is dominated by the 


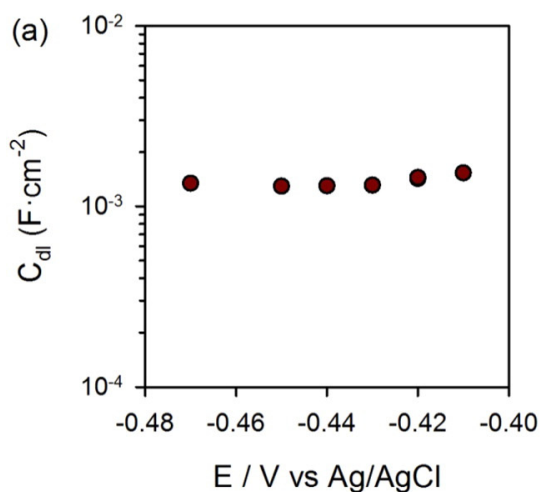

(c)

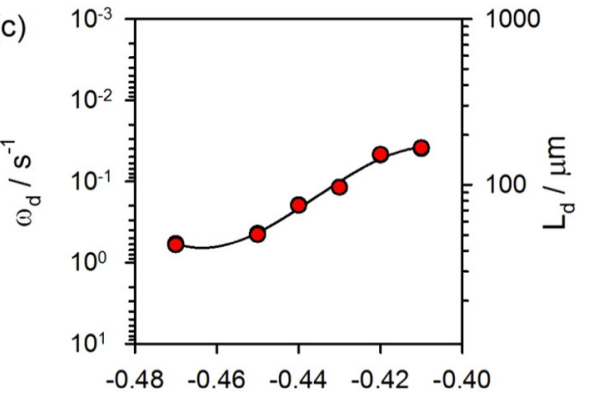

$\mathrm{E} / \mathrm{V}$ vs $\mathrm{Ag} / \mathrm{AgCl}$

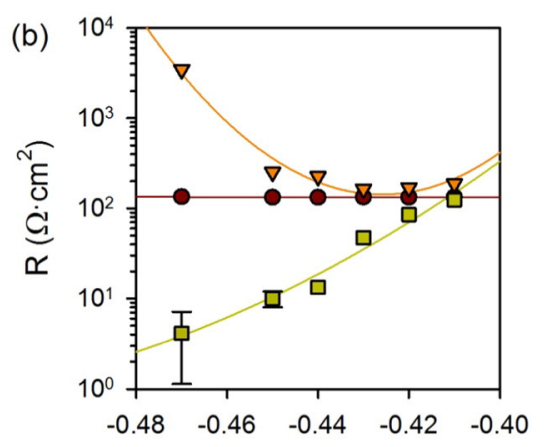

$\mathrm{E} / \mathrm{V}$ vs $\mathrm{Ag} / \mathrm{AgCl}$

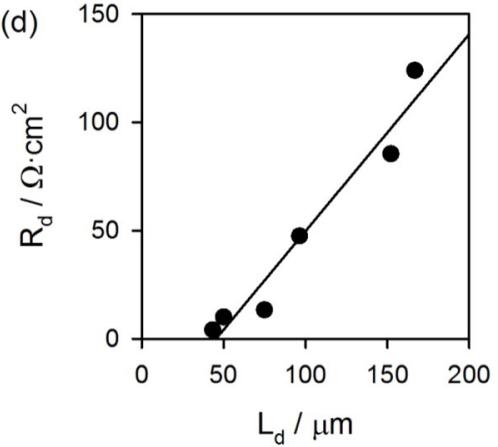

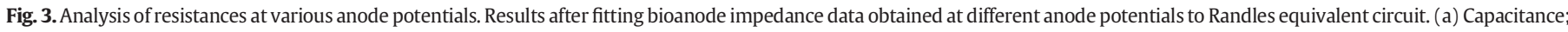

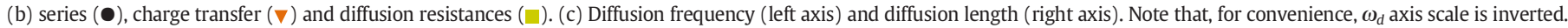
(d) Linear relationship between diffusion length and diffusion resistance.

Butler-Volmer-Monod mechanism [7]. Taking into consideration the general relationship between total resistance, current density and potential given by [17]:

$R_{\text {total }}=\left(\frac{d J}{d V}\right)^{-1}$

with $R_{\text {total }}=R_{S}+R_{c t}+R_{d}$, it is possible to attribute the origin of the Sshape behavior of J-V curve found in Fig. 3(a) to the behavior of the resistances found in Fig. 5(b). Thus, at the most negative anode potentials, the high $R_{c t}$ results in a flattening of the J-V curve, close to the open circuit values. At the most positive anode potentials, the increasing current results in a larger contribution of $R_{d}$ to the total internal resistance, meaning that both the diffusion of substrate (acetata) and products (protons and bicarbonate) towards and from the bioanode, and charge transfer resistance, results in the J-V curve reaching a maximum current. These results match very well with results obtained in a previous paper [5]. It is important to note that the maximum in the J-V curve can be either caused by diffusion limitations, or by a maximum in biological conversion rate, as described in the Butler-Volmer-Monod model.

Impedance data analysis allows to obtain diffusion frequency $\omega_{d}$. With the diffusion coefficient for acetate, $1.1 \times 10^{-5} \mathrm{~cm}^{2} \cdot \mathrm{s}^{-1}$ [18], the length of the diffusion layer in the substrate gradient built around the bioanode may be calculated from the relationship $\omega_{d}=D / L_{d}{ }^{2}$. Results are shown in Fig. 3(c). Note that the scale for $\omega_{d}$ is inverted. Thus, as $\omega_{d}$ increases for more negative potentials, $L_{d}$ diminishes. Fig. 3(d) show the good correlation between $R_{d}$ and $L_{d}$, confirming the validity of data obtained. Maximum diffusion layer in the measured range had $L_{d}=100 \mu \mathrm{m}$; close to the calculated thickness of the boundary layer $(155 \mu \mathrm{m})$ in a previous study [11] with the same bioelectrochemical cell at similar linear flow velocity.

\subsection{Bioanode performance}

To study the performance of bioanodes in more detail, the behavior of the bioanode was studied under different substrate conditions including variations of acetate and buffer concentrations, and $\mathrm{pH}$. The obtained impedance spectra were analyzed in terms of $R_{s}, R_{d}$ and $R_{c t}$. The overall performance of the cell throughout the 2 months period of measurements remained the same, with variations not exceeding $5 \%$.

\subsubsection{Acetate concentration}

24 days after inoculation, the bioanode reached a current density of $1.35 \mathrm{~A} \cdot \mathrm{m}^{-2}$ at the highest controlled potential of $-0.35 \mathrm{~V} \mathrm{vs} \mathrm{Ag} / \mathrm{AgCl}$, in a solution containing $20 \mathrm{mM}$ acetate, $20 \mathrm{mM}$ buffer at $\mathrm{pH} 7$ and with a linear flow velocity of $1.1 \mathrm{~cm} / \mathrm{s}$.

The effect of acetate concentration on bioanode polarization curves can be seen in Fig. 4(a). As expected, the rise in acetate concentration results in an increase in current generated by the bioanode especially at higher potentials, reaching a saturation current density $\left(J_{\text {sat }}\right)$ of $1.35 \mathrm{~A} \cdot \mathrm{m}^{-2}$. This rise, which is linear for low acetate concentrations, is much slower for high concentrations, probably linked to a maximum in biochemical conversion rate. The open circuit anode potential $\left(V_{o c}\right)$ remained nearly constant for the different acetate concentrations.

Note that measurements at $1 \mathrm{mM}$ and $5 \mathrm{mM}$ acetate concentrations provided similar J-V curves, while the measurement at $20 \mathrm{mM}$ resulted in higher current. This can be the result of the bioanode being more developed in time at $20 \mathrm{mM}$, as this condition was tested later on in the experiment. This type of changes in bioanode performance in time although not further elaborated in this study - is important to take into account in this type of bioelectrochemical experiments.

To gain more insight in differences found in the polarization curves, impedance spectroscopy measurements at the different acetate concentrations were performed. In all cases, measurements were done at a fixed anode potential close to $V_{o c}(-0.44 \mathrm{~V}$ vs $\mathrm{Ag} / \mathrm{AgCl})$ to obtain 
(a)

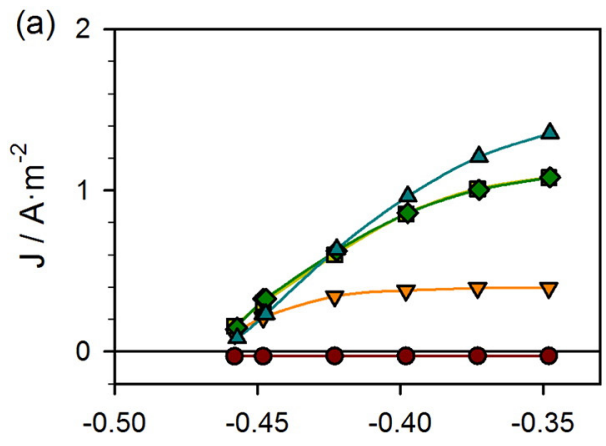

$\mathrm{E} / \mathrm{V}$ vs $\mathrm{Ag} / \mathrm{AgCl}$

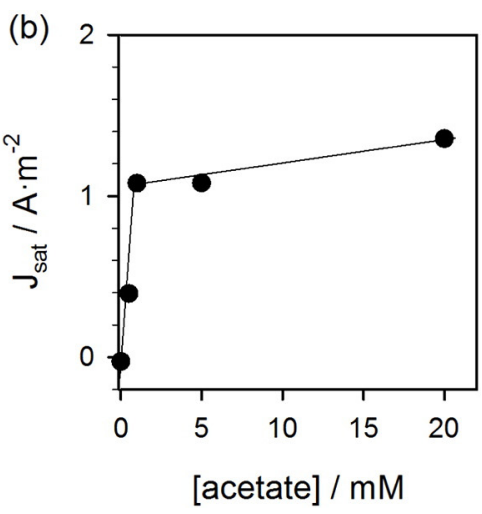

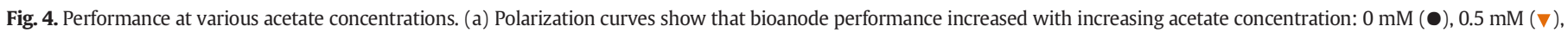
$1 \mathrm{mM}(\mathbf{}), 5 \mathrm{mM}(\downarrow)$, and $20 \mathrm{mM}(\mathbf{\Delta})$. (b) Evolution of current density generated by the bioanode (at $-0.35 \mathrm{~V}$ vs $\mathrm{Ag} / \mathrm{AgCl}$ ) as a function of acetate concentration.

uniform impedance data for our comparison purposes. For the Nyquist plots, see Figure S4 in Supporting Information.

Fig. 5(a) shows the values found for ohmic, mass transport and charge transfer resistances obtained after fitting our data to the Randles circuit in Fig. 2. Capacitance values obtained were constant and close to $1 \mathrm{mF} \cdot \mathrm{cm}^{-2}$, in line with the previous analyses.

Data in Fig. 5 show that the ohmic resistance $R_{S}$ was constant, while both charge and diffusion resistance decreased steeply between $0 \mathrm{mM}$ and $1 \mathrm{mM}$ acetate concentrations, showing a further (less steep) decrease with further increasing acetate concentrations. Except for the case of $0 \mathrm{mM}$ acetate concentration, charge transfer resistance was in all situations roughly a factor 10 higher than the diffusion resistance.

Furthermore, it is observed that $R_{c t}$ decreases with increasing acetate concentration until $1 \mathrm{mM}$ when it shows a less steep decrease. Below these acetate concentrations the drop in $R_{c t}$ with increasing acetate concentration agrees well with simplified charge transfer models as Buttler-Volmer [6] or the bioelectrochemical Buttler-Volmer-Monod [7], which at this potential is given by

$R_{c t}=\frac{A}{k_{r}[A c]} \exp \left[\frac{q\left(V-V_{e q}\right)}{k_{B} T}\right]$ with $A$ a constant, $k_{r}$ the kinetic rate of charge transfer, $[A c]$ the concentration of acetate at the electrode surface, $k_{B}$ Boltzman's constant, $T$ the temperature, $q$ the elementary charge of the electron and the amount $\left(V-V_{e q}\right)$ the overpotential, with $V_{e q}$ the equilibrium potential.

$R_{d}$ continuously decreased with increasing concentration of acetate. In the case of diffusion resistance, the behavior observed is very close to the ideal behavior, which may be approached by [19]:

$R_{d}=\frac{L_{d} k_{B} T}{n^{2} q^{2} S D} \frac{1}{c}$

with $L_{d}$ the diffusion layer length, $S$ the surface of the electrode, $D$ the diffusion coefficient $n$ the number of electrons involved in the charge transfer reaction and $c$ the concentration of the diffusing species (acetate, protons, $\mathrm{D}=9 \times 10^{-5} \mathrm{~cm} \cdot \mathrm{s}^{-2}$ or bicarbonate $\mathrm{D}=$ $\left.1 \times 10^{-5} \mathrm{~cm} \cdot \mathrm{s}^{-2}[18]\right)$.

When representing the inverse of both $R_{c t}$ and $R_{d}$ in Fig. 5(b), the linear behavior of these parameters at low acetate concentration may be better appreciated. A decrease in slope of $R_{d}^{-1}$ is observed in Fig. 5(b) at concentrations of acetate higher than $1 \mathrm{mM}$, but keeping its rising path. The reason for the departure of linearity in Fig. 5(b) could be associated to the decrease in diffusion layer thickness in the proximity of the bioanode, that occurs simultaneously with the increasing (a)

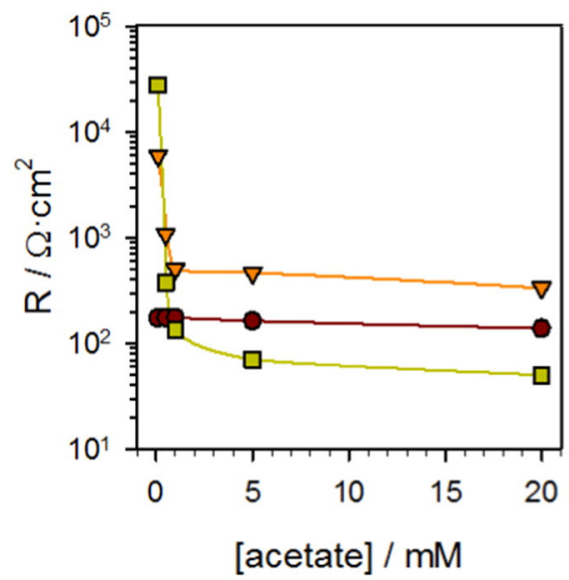

(b)

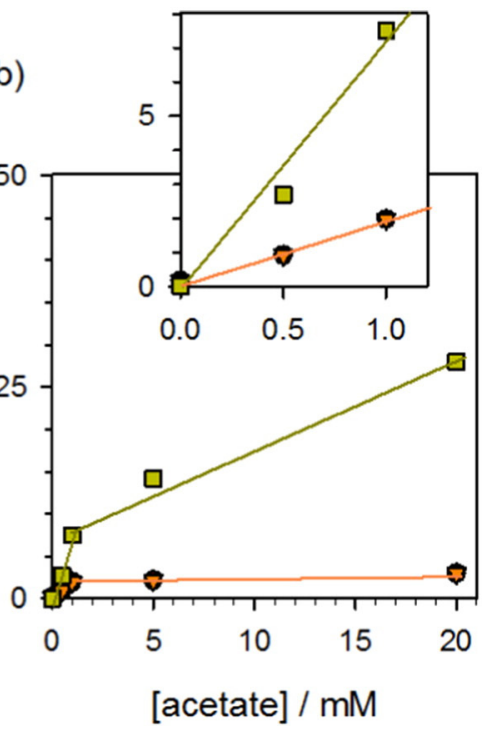

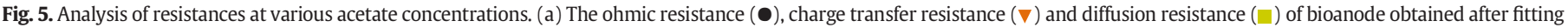

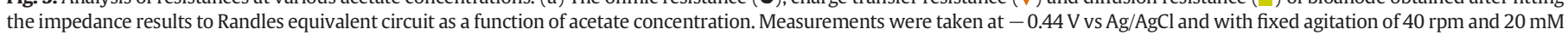
buffer concentration. (b) Representation of the inverse of charge transfer $(\nabla)$ and diffusion resistances ( $\square$ ) for further analysis. 
concentration of acetate. This is in line with the results obtained from diffusion resistance; the diffusion layer decreases with increasing acetate concentration, see Fig. 6(a).

However, the representation of inverse of the product of $R_{d}$ and $L_{d}$ which according to Eq. (4) should depend linearly on acetate concentration departs from this behavior as shown in Fig. 6(b). This result implies that at acetate concentration $>1 \mathrm{mM}$, a change in the process that determines diffusion: at low acetate concentration, current is limited by acetate concentration and therefore, diffusion of acetate towards the biofilm determines $L_{d}$ and $R_{d}$. At high acetate concentration $(>1 \mathrm{mM})$, current is not limited by acetate anymore, but by proton transport away from the biofilm (via buffer species) and thus diffusion of protons away from the biofilm determines $R_{d}$. Thus, at high acetate concentration, $L_{d}$ is not determined anymore by acetate but by buffer concentration. This phenomenon will be further discussed in the following sections on buffer and $\mathrm{pH}$.

The evolution of $\mathrm{R}_{\mathrm{ct}}^{-1}$ with acetate concentration observed in Fig. 5(b) fits very well that of current generation in Fig. 4(b), pointing out a close relation between $R_{c t}$ and $J_{s a t}$, which will be further explored in Section 3.3.

\subsubsection{Buffer concentration}

Without a buffer, the maximum current density was $0.58 \mathrm{~A} \cdot \mathrm{m}^{-2}$, see Fig. 7(a). Increase in buffer concentration resulted in improved performance with a current density up to $3.24 \mathrm{~A} \cdot \mathrm{m}^{-2}$ at $100 \mathrm{mM}$ buffer concentration.

These results show the clear limitation in bioanode performance in the absence of a pH buffer solution, as observed in several other studies $[20,21]$. This is in accordance with other studies reporting on performance limitations attributed to inefficient proton transport away from the bioanode [20]. Interestingly, the curve at $0 \mathrm{mM}$ buffer shows a shift in anode potential, likely due to the different local $\mathrm{pH}$ at the anode, which will be further discussed in the next section on $\mathrm{pH}$.

We performed a further analysis based on fitting the data to the Randles circuit. At low buffer concentrations, however, separate contributions of diffusion and charge transfer resistances were obscured by the high capacitance as discussed before and shown in Fig. 2. At these low buffer concentrations, we found a single deformed arc, see Figure S5 in Supporting information, while at high buffer concentrations ratio between resistances allowed a better determination of diffusion elements. Consequently, data estimated from the fits to equivalent circuit in Fig. 1(b) presented a larger error than in previous measurements. However the main trends of the series, diffusion and charge transfer resistances and that of diffusion frequency are clearly recognizable in Fig. 7(b) and (c). This allows a qualitative analysis of bioanode performance which provided results that are consistent with previous analysis in this paper: i.e., the value for $R_{c t}$ at $20 \mathrm{mM}$ buffer was $340 \Omega \mathrm{cm}^{2}$, similar to the value found in Fig. 5(a) at acetate concentrations $>1 \mathrm{mM}\left(R_{c t} \sim 340 \Omega \mathrm{cm}^{2}\right)$, where acetate was not limiting. Interestingly, the value for $R_{c t}$ in case of buffer limitation ( $0 \mathrm{mM}$ buffer) was $1.1 \mathrm{k} \Omega \mathrm{cm}^{2}$, Fig. 7(b), while the value for $R_{c t}$ in case of acetate limitation ( $0 \mathrm{mM}$ acetate) was $6.0 \mathrm{k} \Omega \mathrm{cm}^{2}$, Fig. 5(a). This suggests that in these extreme cases, acetate limits the performance to a larger extent than buffer.

A first outstanding result is that all three resistances, $R_{S}, R_{d}$ and $R_{c t}$, diminish with increasing amount of buffer. The decrease in the series resistance is the result of the higher conductivity of the solution at higher buffer concentrations. The fast diffusion resistance decrease at low buffer concentration slows down after $20 \mathrm{mM}$ stabilizing its value to $25 \Omega \cdot \mathrm{cm}^{2}$ at $100 \mathrm{mM}$ buffer concentration. Linked to this behavior is the length of the diffusion layer: As shown in Fig. 7(c), above $20 \mathrm{mM}$ buffer, $L_{d}$ is nearly constant $(\sim 65 \mu \mathrm{m})$ with a slight decrease at $100 \mathrm{mM}$ buffer that matches the decrease in $R_{d}$. For low buffer concentrations, the changes in $R_{d}$ cannot be explained by the small variations in $L_{d}$, because $L_{d}$ only shows a clear increase when no buffer is present. So, even though the diffusion length hardly changes, low buffer concentration limits bioanode performance due to proton accumulation.

The continuous decrease of $R_{S}$ with increasing buffer concentration, indicates a better (proton) conductivity in the bulk of the solution, which may be attributed to the presence of the buffer itself or to the higher conductivity of the solution with higher buffer concentration.

With respect to charge transfer resistance, as observed for the different acetate concentrations, the increase in buffer concentration results higher currents in the bioanode and a decrease in $R_{c t}$, which is related to higher bioanode activity.

\subsection{3. $\mathrm{pH}$}

Increasing the $\mathrm{pH}$ from 6 to 8 resulted in a current density increase from $0.7 \mathrm{~A} \cdot \mathrm{m}^{-2}$ to $3.7 \mathrm{~A} \cdot \mathrm{m}^{-2}$ at $-0.35 \mathrm{~V}$ vs $\mathrm{Ag} / \mathrm{AgCl}$, see Fig. 8(a).

In this case, it is important to realize that the theoretical equilibrium anode potential increases by $59 \mathrm{mV}$ for every unit decrease in $\mathrm{pH}$ according to Nernst law [1]. Following this, the J-V curves in Fig. 8(a) should be better compared by correcting the potential $59 \mathrm{mV}$ to the right for the $\mathrm{pH} 8$ curve and to $59 \mathrm{mV}$ the left for the $\mathrm{pH} 6$ curve for a good comparison with pH 7, as in Fig. 8(b). After these potential shifts, it is hard to see such clear difference in bioanode performance for $\mathrm{pH} 6$ and 7, compared to the situation where the anode potential was not corrected. The improvement in bioanode performance at $\mathrm{pH} 8 \mathrm{com}$ pared to $\mathrm{pH} 7$ is still clearly visible.

Parameters obtained from the fitting of impedance data (see Nyquist plots in Figure S6 of Supporting information) plotted in Fig. 9(a) are also affected by the potential shift due to differences in $\mathrm{pH}$, which makes the analysis of data more difficult. This effect is less important for diffusion resistance than for charge transfer resistance. For pH 6, however, the estimation of $R_{d}$ contains a large error due to the coupling of diffusion and charge transfer processes (that are associated to values of $C_{d l}, R_{d}$ and $R_{c t}$ ), and a reliable value of $\omega_{d}$ could not be determined. For $\mathrm{pH} 7$ and $\mathrm{pH} 8$, diffusion resistance seems to be unaffected by the change in $\mathrm{pH}$.
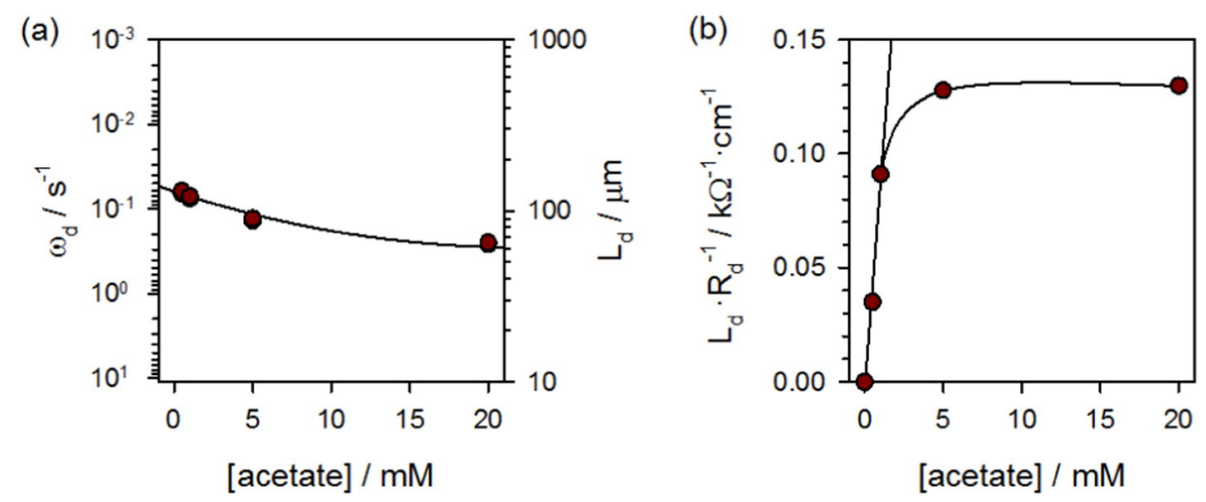

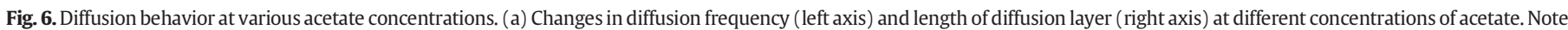
that for convenience, scale in the frequency axis is inverted. (b) Non-linear behavior of inverse of the product of diffusion resistance and diffusion length vs acetate concentration. 
(a)

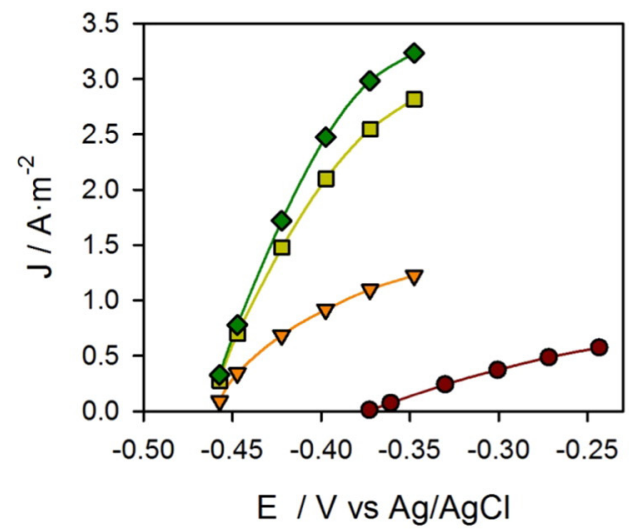

(b)

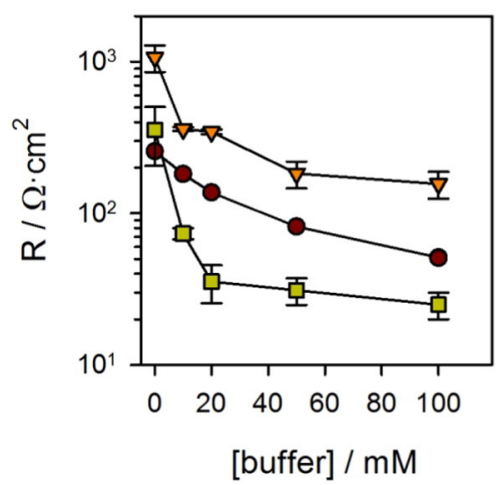

(c)

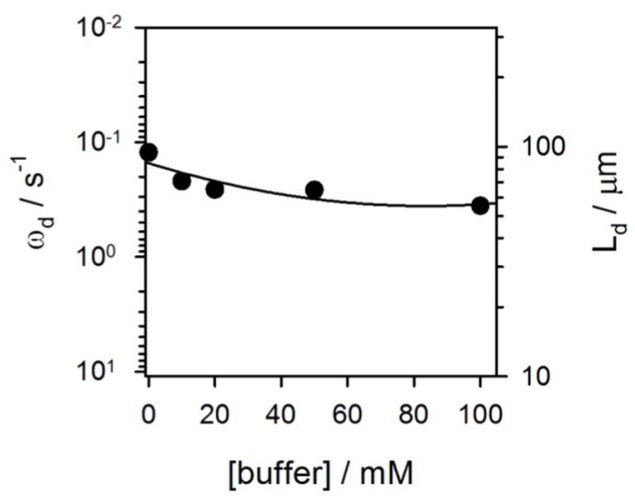

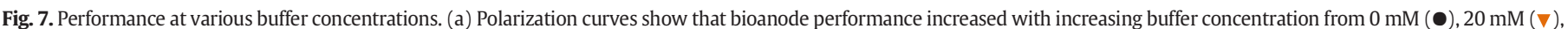

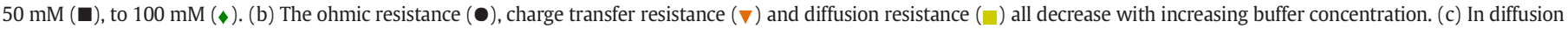
frequency (left axis) and length of diffusion layer (right axis) as a function of buffer concentration.

At $\mathrm{pH} 8, \omega_{d}$ decreased compared to $\mathrm{pH} 7$, resulting in a larger $L_{d}$ in our estimation (Fig. 9(b)). Because at similar $\omega_{d,} L_{d}$ can only change when $D$ changes, according to $\omega_{d}=D / L_{d}{ }^{2}$, the change in $L_{d}$ can be explained by an increase in effective diffusion coefficient at $\mathrm{pH} 8$ compared to $\mathrm{pH}$ 7. This indicates that proton transport is influencing the effective diffusion coefficient in the electrolyte, while diffusion resistance of acetate remains constant.

Comparison of the result for $\mathrm{R}_{\mathrm{ct}}$ at the different $\mathrm{pH}$ values is difficult, because the $\mathrm{R}_{\mathrm{ct}}$ was determined at a fixed anode potential and the (theoretical) anode potential is $\mathrm{pH}$ dependent. For $\mathrm{pH} 6$, this anode potential represents a different part of the polarization curve than at $\mathrm{pH} 7$ and 8 , where Monod limitations become apparent, and therefore changes in $R_{c t}$ are not directly comparable. However, data from $\mathrm{pH} 7$ and 8 can be compared without assuming large deviations from real values because

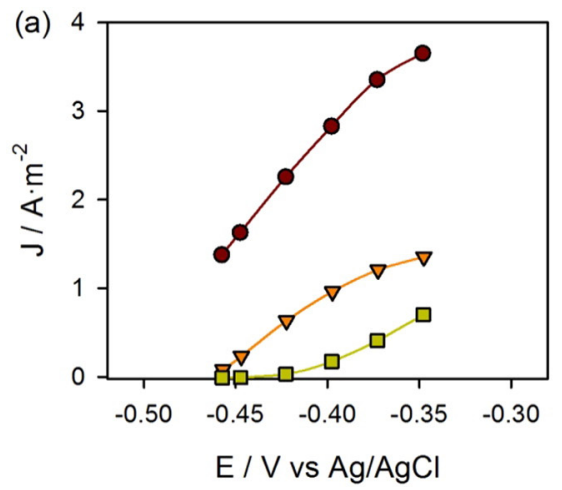

the polarization curves are similar and not limited by Monod kinetics. With these preventions, we can see in Fig. 9(b) that $R_{c t}$ diminishes for the bioanode at higher $\mathrm{pH}$, which resulted in higher current density. In this respect, it is interesting to note that the effectiveness of the buffer is also highly dependent on $\mathrm{pH}$, with phosphate buffer having an optimal effectiveness at its $\mathrm{pK}_{\mathrm{a}}$ value of 7.2 [22]. The value for $R_{d}$ being higher at pH 6 than at pH 7 and 8 can therefore also be an effect of lower effectiveness, and thus buffer capacity, of the phosphate buffer at this low $\mathrm{pH}$ - although buffer concentration was the same.

\subsection{Advantages of distinguishing $R_{c t}$ and $R_{d}$}

The advantages of impedance measurements in addition to $\mathrm{J}-\mathrm{V}$ curves is, that the charge transfer and diffusion resistances can be

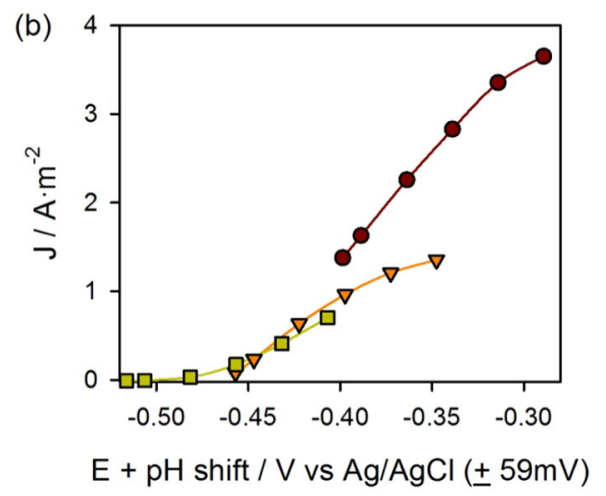

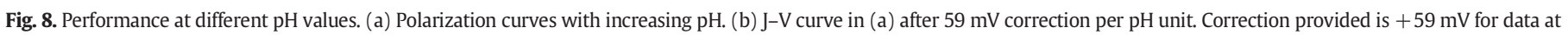
$\mathrm{pH} 8$ and $-59 \mathrm{mV}$ for data at pH 6. Solution composition was kept at with $20 \mathrm{mM}$ of acetate and $20 \mathrm{mM}$ of buffer. pH $6(\square), \mathrm{pH} 7(\boldsymbol{\nabla}), \mathrm{pH} 8(\bullet)$. 
(a)

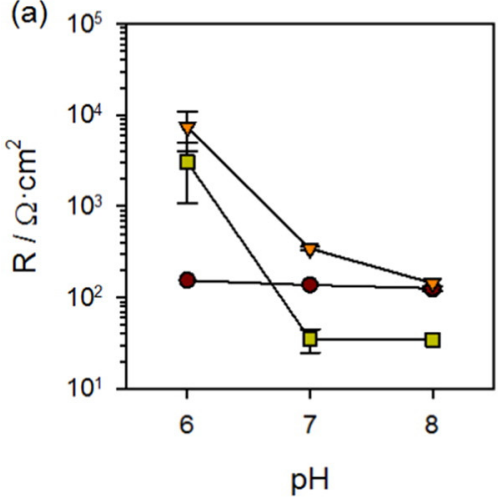

(b)

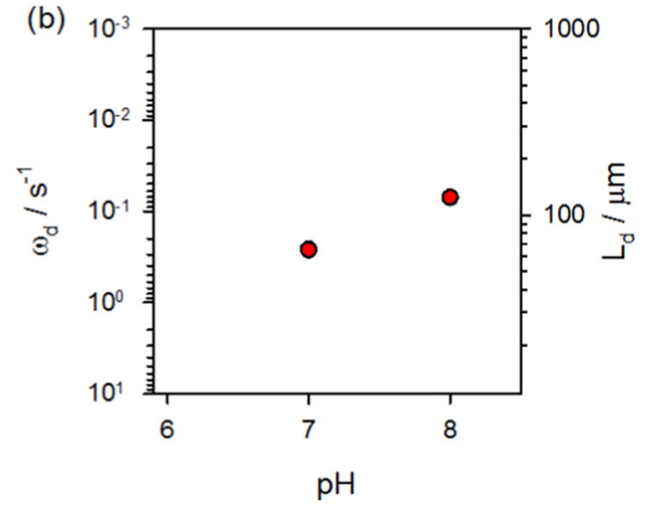

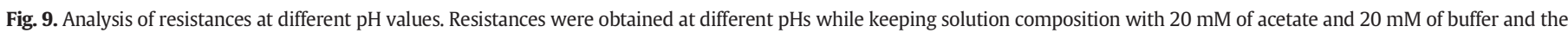

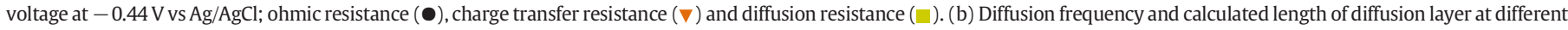
$\mathrm{pH}$.

separated. This enables more detailed understanding of the factors that determine bioanode performance.

In the previous sections, we found a clear relationship between the charge transfer resistance at $-0.44 \mathrm{~V}$ vs $\mathrm{Ag} / \mathrm{AgCl}$ and the saturation current density - maximum current density measured in the polarization curves at $-0.35 \mathrm{~V}$ vs $\mathrm{Ag} / \mathrm{AgCl}$. The origin of this behavior is associated to the fact that for this potential range, the polarization curve follows a Buttler-Volmer behavior. Together with definition of $R_{c t}$ given in Eq. (4) this produces a very simple relationship between $R_{c t}$ at values close to open circuit $(-0.44 \mathrm{~V}$ vs $\mathrm{Ag} / \mathrm{AgCl}$ ) and saturation current (at $-0.35 \mathrm{~V}$ vs $\mathrm{Ag} / \mathrm{AgCl}$ )

$J_{\text {sat }}=\frac{k_{B} T}{q \beta} \frac{1}{R_{c t}}$

with $\beta$ the transfer factor in Butler-Volmer equation, which has been already observed in other electrochemical systems as dye solar sensitized solar cells or also polymeric solar cells, $[23,24]$.

Data of all our bioanodes are collected in Fig. 10, showing a good agreement with Eq. (6) and and yielding $\beta=0.50$, a value typically obtained for the transfer factor of metals and some semiconductors.

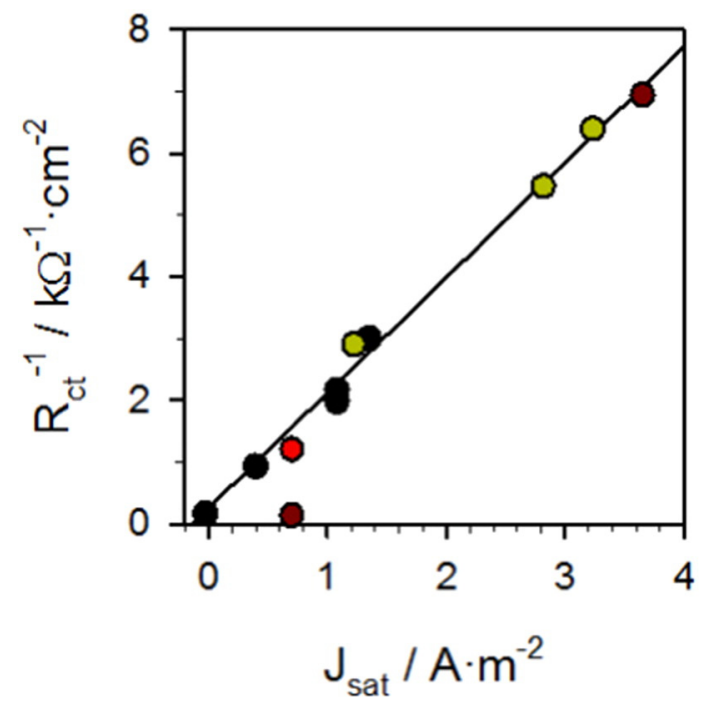

Fig. 10. Relationship between the inverse of charge transfer resistance and bioanode saturation current. Included are all samples with different $\mathrm{pH}$ (red), acetate (black) and buffer (yellow) concentrations. Data at $\mathrm{pH} 6$ (clear red) has been obtained considering $\mathrm{R}_{\mathrm{ct}}$ values at the corrected (shifted) potential of $-0.44 \mathrm{~V}$. (For interpretation of the references to color in this figure legend, the reader is referred to the web version of this article.)
The fact that this relationship is found, confirms that the behavior of the bioanode at a potential of $-0.44 \mathrm{~V} \mathrm{vs} \mathrm{Ag} / \mathrm{AgCl}$ is in accordance with the Butler-Volmer equation. Indeed, bioanode performance at values close to open circuit is expected not to be limited by Monod kinetics, because this limitation becomes apparent only at higher overpotentials, following the bioelectrochemical models $[7,9]$. Further exploration and discussion is needed to analyze the specific meaning of this linear relationship in BESs and to analyze to which extent the knowledge already present in the field of solar cells can be used to better analyze and understand bioanode performance.

In summary, the use of impedance analysis allows separation of charge transfer and diffusion resistances of the cell at the operating potentials of the cells from other effects such capacitance or other series resistances. Diffusion frequency, and so the calculated diffusion length, helps to complete the analysis provided by resistances. This enables a more detailed understanding of the factors that determine bioanode performance.

Large capacitances found have been associated to graphite electrode. Despite the high capacitances, our experimental approach in combination with impedance analyses gave consistent results.

\section{Conclusions}

Experimental conditions for the operation of the bioanode in a microbial fuel cell have been systematically studied in combination with EIS to analyze the main factors affecting bioanode performance. Best operating conditions were obtained with $100 \mathrm{mM}$ buffered solutions and $\mathrm{pH}$ 8. Effect of acetate concentration (tested at $\mathrm{pH} 7$ and $20 \mathrm{mM}$ buffer) was very pronounced below $1 \mathrm{mM}$. Increase of acetate above $1 \mathrm{mM}$ had little effect on current generation.

A qualitative description of the parameters determining the performance of the cell is provided. Origin of large capacitances $\left(1 \mathrm{mF} \cdot \mathrm{cm}^{-2}\right)$ found in the bioanode was associated to the graphite anode used as supporting electrode for the microbial culture. The effect of the different experimental conditions on solution, charge transfer and diffusion resistances was described and used to understand the limitations of bioanode performance.

At anode potentials close to open circuit, charge transfer resistance was the main contribution to total resistance, except for acetate concentrations lower than $1 \mathrm{mM}$, and charge transfer was thus the parameter determining the behavior of J-V curve. Values of solution and diffusion resistance have a relatively low importance as the current flowing through the cell is (still) low.

Separation of charge and diffusion processes can give a starting point for new analyses. For example, a linear dependence on the saturation current generated by the bioanode and inverse charge transfer 
resistance at values close to open circuit was found, which needs further exploration.

\section{Acknowledgments}

We thank David Strik for his help with the design of the experiments.

The authors wish to acknowledge funding from the European Union Seventh Framework Programme (FP7/2012-2016) project 'Bioelectrochemical systems for metal production, recycling, and remediation' under grant agreement no. 282970.

AtH is supported by a NWO VENI grant no. 13631.

OS was supported by the French environmental agency ADEME, by the Region Bretagne and by Rennes Metropole when doing the experiments.

This work was performed in the cooperation framework of Wetsus, Centre of Excellence for Sustainable Water Technology (www.wetsus. $\mathrm{nl}$ ). Wetsus is co-funded by the Dutch Ministry of Economic Affairs and Ministry of Infrastructure and Environment, the European Union Regional Development Fund, the Province of Fryslân, and the Northern Netherlands Provinces.

\section{Appendix A. Supplementary data}

Supplementary data to this article can be found online at http://dx. doi.org/10.1016/j.bioelechem.2015.04.002.

\section{References}

[1] B.E. Logan, B. Hamelers, R. Rozendal, U. Schröder, J. Keller, S. Freguia, P. Aelterman, W. Verstraete, K. Rabaey, Microbial fuel cells: methodology and technology, Environ. Sci. Technol. 40 (17) (2006) 5181-5192.

[2] H.V.M. Hamelers, A. Ter Heijne, T.H.J.A. Sleutels, A.W. Jeremiasse, D.P.B.T.B. Strik C.J.N. Buisman, New applications and performance of bioelectrochemical systems, Appl. Microbiol. Biotechnol. 85 (6) (Feb. 2010) 1673-1685.

[3] X. Dominguez-Benetton, S. Sevda, K. Vanbroekhoven, D. Pant, The accurate use of impedance analysis for the study of microbial electrochemical systems, Chem. Soc. Rev. 41 (21) (Nov. 2012) 7228-7246.

[4] D.P.B.T.B. Strik, A. Ter Heijne, H.V.M. Hamelers, M. Saakes, C.J.N. Buisman, Feasibility Study on Electrochemical Impedance Spectroscopy for Microbial Fuel Cells: Measurement Modes \& Data Validation, ECS Transactions, 13 (21) (2008) 27-41.

[5] A. Ter Heijne, O. Schaetzle, S. Gimenez, F. Fabregat-Santiago, J. Bisquert, D.P.B.T.B. Strik, F. Barriere, C.J.N. Buisman, H.V.M. Hamelers, Identifying charge and mass transfer resistances of an oxygen reducing biocathode, Energy Environ. Sci. 4 (12) (2011) 5035-5043.
[6] A.J. Bard, L.R. Faulkner, Electrochemical Methods, Fundamentals and Applications, Second edition John Wiley \& Sons, Weinheim, 2001.

[7] H.V.M. Hamelers, A. Ter Heijne, N. Stein, R.A. Rozendal, C.J.N. Buisman, ButlerVolmer-Monod model for describing bio-anode polarization curves, Bioresour. Technol. 102 (1) (Jan. 2011) 381-387.

[8] C.I. Torres, A.K. Marcus, P. Parameswaran, B.E. Rittmann, Kinetic experiments for evaluating the Nernst-Monod model for anode-respiring bacteria (ARB) in a biofilm anode, Environ. Sci. Technol. 42 (17) (Sep. 2008) 6593-6597.

[9] A. Kato Marcus, C.I. Torres, B.E. Rittmann, Conduction-based modeling of the biofilm anode of a microbial fuel cell, Biotechnol. Bioeng. 98 (6) (Dec. 2007) 1171-1182.

[10] A. Ter Heijne, D.P.B.T.B. Strik, H.V.M. Hamelers, C.J.N. Buisman, Cathode potential and mass transfer determine performance of oxygen reducing biocathodes in microbial fuel cells, Environ. Sci. Technol. 44 (18) (Sep. 2010) 7151-7156.

[11] A. ter Heijne, H.V.M. Hamelers, M. Saakes, C.J.N. Buisman, Performance of nonporous graphite and titanium-based anodes in microbial fuel cells, Electrochim. Acta 53 (18) (Jul. 2008) 5697-5703.

[12] G.J. Brug, A.L.G. van den Eeden, M. Sluyters-Rehbach, J.H. Sluyters, The analysis of electrode impedances complicated by the presence of a constant phase element, J. Electroanal. Chem. Interfacial Electrochem. 176 (1-2) (Sep. 1984) 275-295.

[13] T. Piao, S.M. Park, C.H. Doh, S.I. Moon, Intercalation of lithium ions into graphite electrodes studied by AC impedance measurements, J. Electrochem. Soc. 146 (1999) 2794-2798.

[14] J.E. Fischer, H.J. Kim, Elastic effects in intercalation compounds: comparison of lithium in graphite and TiS2, Phys. Rev. B 35 (1987) 3295-3298.

[15] S.A. Safran, D.R. Hamann, Long-range elastic interactions and staging in graphite intercalation compounds, Phys. Rev. Lett. 42 (1979) 1410-1413.

[16] Q. Guo, V.R. Subramanian, J.W. Weidner, R.E. White, Estimation and diffusion coefficient of lithium in carbon using AC impedance technique, J. Electrochem. Soc. 149 (2002) 318.

[17] F. Fabregat-Santiago, J. Bisquert, E. Palomares, L. Otero, D. Kuang, S.M. Zakeeruddin, M. Grätzel, Correlation between photovoltaic performance and impedance spectroscopy of dye-sensitized solar cells based on ionic liquids, J. Phys. Chem. C 111 (2007) 6550-6560.

[18] J.E. Dykstra, P.M. Biesheuvel, H. Bruning, A. Ter Heijne, Theory of ion transport with fast acid-base equilibrations in bioelectrochemical systems, Phys. Rev. E 90 (1) (Jul. 2014) 013302

[19] J. Bisquert, G. Garcia-Belmonte, F. Fabregat-Santiago, P.R. Bueno, Theoretical models for ac impedance of finite diffusion layers exhibiting low frequency dispersion, J. Electroanal. Chem. 475 (2) (1999) 152-163.

[20] C.I. Torres, A. Kato Marcus, B.E. Rittmann, Proton transport inside the biofilm limits electrical current generation by anode-respiring bacteria, Biotechnol. Bioeng. 100 (5) (Aug. 2008) 872-881.

[21] T.H. Pham, P. Aelterman, W. Verstraete, Bioanode performance in bioelectrochemical systems: recent improvements and prospects, Trends Biotechnol. 27 (3) (Mar. 2009) $168-178$.

[22] A.W. Jeremiasse, H.V.M. Hamelers, J.M. Kleijn, C.J.N. Buisman, Use of biocompatible buffers to reduce the concentration overpotential for hydrogen evolution, Environ. Sci. Technol. 43 (17) (Sep. 2009) 6882-6887.

[23] P.P. Boix, A. Guerrero, L.F. Marchesi, G. Garcia-Belmonte, J. Bisquert, Current-voltage characteristics of bulk heterojunction organic solar cells: connection between light and dark curves, Adv. Energy Mater. 1 (6) (2011) 1073-1078.

[24] S.R. Raga, E.M. Barea, F. Fabregat-Santiago, Analysis of the origin of open circuit voltage in dye solar cells, J. Phys. Chem. Lett. 3 (12) (2012) 1629-1634. 